\title{
Recomendaciones de la Comisión de Broncoscopía y Neumología Intervencionista de la Sociedad Chilena de Enfermedades Respiratorias en el uso de la Broncoscopía y toma de muestras en pacientes con sospecha o diagnóstico de infección por COVID-19
}

\author{
ALFREDO JALILIE E.*,PAULA BARCOS M.*, ARTURO MORALES S.*, \\ MACARENA RODRIGUEZ V.* y DAVID LAZO P.**
}

Statement of bronchoscopy and interventional pneumology commission of the Chilean Society of Respiratory Diseases on bronchoscopy and specimen collection in patients with suspected or confirmed COVID-19

Considerando la pandemia global a causa del nuevo Coronavirus 19 (COVID-19) y el aumento progresivo de casos en nuestro país, la Comisión de Broncoscopía y Neumología Intervencionista de la SER ha redactado este documento sobre el uso seguro y eficiente de los procedimientos broncoscópicos en pacientes con sospecha o diagnóstico confirmado de COVID-19. Dada la fase actual de contagio (Etapa 4) todo sujeto sometido a broncoscopía debe ser considerado como caso sospechoso (independiente de síntomas) y manejado con precauciones respiratorias y de contacto dado la liberación de aerosoles que se generan durante el examen.

El objetivo principal de estas recomendaciones es proporcionar normas de seguridad durante esta pandemia, dirigidas al paciente, al personal de salud y a la comunidad.

Este documento se basa en las recomendaciones del Center for Diseases Control (CDC), American Association of Bronchology and Interventional Pulmonology (AAIBP) y la Asociación Española de Endoscopia Respiratoria (AEER).

\section{Recomendaciones generales de recolección} de muestras en pacientes con sospecha diagnóstica de infección por COVID-19

1. El método diagnóstico de elección es el muestreo nasofaríngeo y orofaríngeo con tórula diseñada para tal propósito.
2. La toma de muestra de vía aérea superior se recomienda en pacientes con sospecha de infección por COVID-19 según las indicaciones establecidas por el MINSAL y las Sociedad Chilena de Infectología.

3. No se recomienda el esputo inducido por el riesgo de generar aerosoles infectantes.

4. La broncoscopía debe tener un rol LIMITADO en estos pacientes pues genera aerosoles infectantes. Debe ser CONSIDERADA en casos de pacientes intubados con alta sospecha diagnóstica en quienes las muestras de vía aérea superior hayan sido negativas o cuando exista un diagnóstico alternativo, que pudiese influir directamente en la conducta clínica.

5. En el caso que la broncoscopía deba ser realizada para confirmación diagnóstica de $\mathrm{CO}$ VID, se recomienda recolectar un mínimo de 2 a $3 \mathrm{ml}$ en frasco estéril antireflujo.

6. En la realización de la broncoscopía sólo debe estar el personal indispensable (broncoscopista, enfermera y técnico en broncoscopia), por el tiempo mínimo necesario:

Siempre se debe tener presente que la broncoscopía es un procedimiento capaz de aerolizar secreciones de vía aérea, por lo que frente a un paciente con sospecha o diagnóstico de $\mathrm{CO}$ VID-19 existe riesgo de contagio. Se entiende luego que las broncoscopias no urgentes deban ser pospuestas y que sólo se deban realizar las

* Comisión de Broncoscopía y Neumología Intervencionista de la Sociedad Chilena de Enfermedades Respiratorias. 
Tabla 1. Propuesta de realización de broncoscopía en contexto de pandemia por COVID-19 (no realizar broncoscopías no urgentes)

\begin{tabular}{|c|c|c|}
\hline Broncoscopia de emergencia & Broncoscopia de urgencia & Broncoscopia no urgente \\
\hline $\begin{array}{l}\text { Estenosis de vía aérea moderada o } \\
\text { severa sintomática }\end{array}$ & $\begin{array}{l}\text { Masa pulmonar sospechosa de } \\
\text { cáncer pulmonar }\end{array}$ & $\begin{array}{l}\text { Estenosis leve de vía aérea traqueal } \\
\text { o bronquial }\end{array}$ \\
\hline $\begin{array}{l}\text { Obstrucción de vía aérea central } \\
\text { por masa endoluminal o tapones } \\
\text { mucosos }\end{array}$ & $\begin{array}{l}\text { Adenopatías mediastínicas o hiliares } \\
\text { sospechosas de malignidad }\end{array}$ & $\begin{array}{l}\text { Aspiración de secreciones, aseo } \\
\text { bronquial }\end{array}$ \\
\hline Hemoptisis masiva & $\begin{array}{l}\text { Atelectasia lobar o de hemitórax } \\
\text { completo }\end{array}$ & $\begin{array}{l}\text { Alta sospecha de sarcoidosis sin } \\
\text { necesidad de tratamiento de urgencia }\end{array}$ \\
\hline \multirow{4}{*}{$\begin{array}{l}\text { Migración de stent en vía } \\
\text { aérea }\end{array}$} & Aspiración de cuerpo extraño & Enfermedad pulmonar intersticial \\
\hline & $\begin{array}{l}\text { Hemoptisis leve a moderada } \\
\text { (no expectoración hemoptoica) }\end{array}$ & Detección de micobacterias atípicas \\
\hline & $\begin{array}{l}\text { Lavado broncoalveolar en pacientes } \\
\text { inmunosuprimidos }\end{array}$ & Tos crónica \\
\hline & & Evaluación de traqueobroncomalacia \\
\hline
\end{tabular}

broncoscopias de emergencia o urgencia (Tabla 1), o las necesarias para confirmar COVID si test no invasivos han sido negativos.

7. El buen uso de elementos de protección personal minimiza el riesgo de contagio.

\section{Preparación del personal en caso necesario de broncoscopía en pacientes con sospecha diagnóstica de COVID-19.}

1. El paciente debe estar en una habitación con presión negativa de estar disponible, debe estar en aislamiento respiratorio y de contacto.

2. A todos los pacientes (excepto en ventilación mecánica), se les debe poner mascarilla quirúrgica para evitar la emisión de gotas en caso de tos, antes y después del procedimiento.

3. En caso de pacientes en Ventilación invasiva, se debe usar conector swivel para disminuir el riesgo de escape de aerosoles generados por la broncoscopía y pinzar el tubo orotraqueal en cada desconexión de circuito.

4. No se debe realizar broncoscopias a pacientes en ventilación no invasiva; se debe intubar a pacientes para el examen.

5. Todo el personal debe usar Equipo de Protección Personal (PPE) que incluye gorro, guantes, delantal desechable, protección respiratoria y escudos faciales. La protección respiratoria puede ser un respirador purificante de aire o una mascarilla purificadora de aire tipo N95, FFP2 o FFP3. Evitar el contacto con aerosoles mediante estas medidas es de vital importancia.

6. Se deben seguir las instrucciones precisas en cuanto a la forma de colocación y retiro de los EPP (Anexo 1).

7. Guías internacionales recomiendan el uso de broncoscopios de un solo uso, desechables.

8. En caso de no contar con broncoscopios desechables, se debe seguir el protocolo de uso de detergente enzimático al lado de cama de enfermo y luego desinfección de alto nivel o esterilización.

\section{Indicaciones de broncoscopía en pacientes con sospecha o COVID-19 confirmado}

La broncoscopía debe ser evitada en pacientes con COVID-19. El rol de la broncoscopia en estos pacientes es confirmar el diagnóstico en pacientes con alta sospecha de COVID-19 que tengan aspirado nasofaringeo y orofaringeo negativos, en pacientes con sospecha de diagnósticos alternativos o en casos de urgencias y emergencias broncoscopicas. 
Anexo 1. Uso de elementos de protección personal

\section{Instalación de elementos de protección}

1. Lavado de manos

2. Instalación de pechera manga larga

3. Instalación de mascarilla N95

4. Instalación de escudo facial

5. Instalación de guantes

6. Se sugiere, en norma ministerial que al final del proceso, se utilice un segunda pechera desechable impermeable (Circular C37 Número 1 del 18 Marzo 2020).

(Personas con pelo largo deben asegurarlo antes de instalar los elementos de protección para que no roce la cara)

Retirado (a más de 1,5 $\mathrm{m}$ de paciente, ideal en exclusa de pieza)

1. Eliminar guantes y bata (considerar zona externa de guantes y bata como contaminado y retirar sin contaminar piel). En caso de doble pechera eliminar la externa junto a guantes y luego la interna

2. Lavarse las manos

3. Sacar escudo fácil (zona externa está contaminada, sacar de atrás hacia adelante)

4. Lavarse las manos

5. Sacar mascarilla N95 (sin tomarla de frente sino por elásticos traseros, primero inferior y luego superior, manteniendo siempre tracción entre retiro de ambos elásticos para que este lejos de la cara).

6. Lavarse las manos

\section{Bibliografía}

1.- AAIBP statement on the use of bronchoscopy and respiratory specimen collection in patients with suspected or confirmed COVID-19 infection.

2.- Sitio web CDC: https://www.cdc.gov/coronavirus/2019$\mathrm{nCov} / \mathrm{hcp} / \mathrm{clinical}$-criteria.html
3.- Recomendaciones de consenso SEPAR y AEER sobre el uso de la broncoscopía y la tomas de muestras de la vía respiratoria en pacientes con sospecha o con infección confirmada por COVID19.

4.- Recomendaciones de uso de elementos de protección personal (EPP) para trabajadores de la Salud. Colegio Médico de Chile y Sociedad Chilena de Infectología.

Correspondencia a:

Dr. Alfredo Jalilie Elías

Comisión de Broncoscopía y Neumología

Intervencionista

Sociedad Chilena de Enfermedades Respiratorias

Email: ajalilie@clinicasantamaria.com 